\title{
ARTICLE OPEN \\ NPTX2 is a key component in the regulation of anxiety
}

\author{
Simon Chang ${ }^{1,2}$, Philane Bok ${ }^{1}$, Ching-Yen Tsai ${ }^{3}$, Cheng-Pu Sun ${ }^{4}$, Hsuan Liu ${ }^{1,5}$, Jan M. Deussing $\mathbb{C}^{2}$ and Guo-Jen Huang ${ }^{1,6,7}$
}

Anxiety disorders significantly impair quality of life. However, limited knowledge of the underlying mechanisms impedes the development of effective therapeutics. Previous studies have suggested that the expression of the Nptx2 gene is associated with anxiety, but the neurobiological processes underlying this association remain unclear. We generated multiple mouse models with knockout or overexpression of Nptx2 in specific brain regions and during different developmental stages to assess anxiety, adult neurogenesis, and glucocorticoid-related gene expression. Our results provide evidence that Nptx2 expression in the adult hippocampus regulates anxiety in mice. Eliminating Nptx2 expression in either the developing mouse brain or in adulthood leads to increased anxiety levels. The increase in anxiety was evident in hippocampus-specific Nptx2 knockout mice, but not in an amygdala specific knockouts. Gene expression analysis revealed increased expression of glucocorticoid receptor target genes in Nptx2 knockout mice after acute stress. Overexpression of Nptx2 in the hippocampus alleviates stress-induced anxious behaviors and reverses the changes in expression of glucocorticoid receptor related genes. In conclusion, we demonstrate that Nptx 2 in the hippocampus performs a critical role in modulating anxiety, hippocampal cell proliferation, and glucocorticoid receptor related gene expression. Our results suggest Nptx2 may be a potential target for anxiolytic therapeutics.

Neuropsychopharmacology (2018) 43:1943-1953; https://doi.org/10.1038/s41386-018-0091-z

\section{INTRODUCTION}

Anxiety disorders are highly prevalent and have the capacity to severely perturb quality of life. Unfortunately, the underlying molecular mechanisms that regulate normal and pathological mood states are currently poorly understood. We previously reported that Nptx2 (also called NARP, neuronal activity-regulated pentraxin) is the most significantly upregulated transcript in the hippocampus in mice treated with either an antidepressant or exercise [1]. Other studies have shown that NPTX2 is altered in pathological states such as Alzheimer's disease [2] and schizophrenia [3]. To date, not much is known about the role of NPTX2 in anxiety.

In situ hybridization (ISH) images from the Allen Brain Atlas (http://brain-map.org/) show that the gene is expressed in limbic and cortical regions. Nptx2, also named Narp (neuronal activityregulated pentraxin) is a secreted protein, first identified in venom research [4]. NPTX2 protein is localized specifically to excitatory synapses in both pre- and postsynaptic compartments in the adult brain to induce the aggregation of neuronal aamino-3-hydroxy-5-methyl-4-isoxazolepropionic acid receptors (AMPA receptors) [5]. NPTX2 interacts with other members of the pentraxin family, which include NPTX 1 and NPTXR/3 to form complexes that bind to AMPA receptors $[6,7]$. Evidence shows NPTX2 accumulates on parvalbumin-expressing interneurons to regulate homeostatic scaling of excitatory synapses [8]. Mice without NPTX2/NPTXR exhibit profound loss of GluA4 and AMPA receptor function in parvalbumin-expressing neurons, suggesting NPTXs plays an essential role in inhibition/excitation balance
[9]. Interventions that influence emotional responses also regulate Nptx2 expression. Long-term treatment with antidepressants, electroconvulsive seizure, or brain-derived neurotrophic factor (BDNF) infusion all improve emotional status, as well as increase the expression of Nptx2 in the hippocampus [10-14].

In this study, we generated loxP-flanked Npxt2 knockout (KO) mice to examine the role of Nptx2 on anxiety. This manipulation enabled precise elimination of Nptx2 expression in a spatially and/ or temporally controlled manner. Our data suggest that hippocampal Nptx2 plays a critical role in regulating anxiety, progenitor cell proliferation, and the expression of genes that underlie the glucocorticoid response. Thus, our study provides strong evidence that hippocampal Nptx2 regulates anxiety and reveals it as a potential target for novel treatments for stress-related anxiety disorders.

\section{MATERIALS AND METHODS}

Animals

Experiments were performed on sex balanced cohorts of nineweek-old Nptx2 KO and wild- type littermate mice. Nptx2 floxed mice were generated from ES cells from IMPC (International Mouse Phenotyping Consortium). Sox1::Cre mice (Acc No. CDB0525K) were genotyped as described previously [15]. CAG:: $\mathrm{CreER}^{T 2}$ transgenic mice were purchased from Jax Lab (Stock number: 004682). Behavioral tests were conducted during the dark phase. Mice were housed in a 12:12 h light-dark cycle at $22^{\circ}$

\footnotetext{
${ }^{1}$ Department and Graduate Institute of Biomedical Sciences, College of Medicine, Chang Gung University, Taoyuan, Taiwan; ${ }^{2}$ Department of Stress Neurobiology and Neurogenetics, Max Planck Institute of Psychiatry, Munich, Germany; ${ }^{3}$ Institute of Molecular Biology, Academia Sinica, Taipei, Taiwan; ${ }^{4}$ Institute of Biomedical Sciences, Academia Sinica, Taipei, Taiwan; ${ }^{5}$ Molecular Medicine Research Center, Chang Gung University, Taoyuan, Taiwan; ${ }^{6}$ Healthy Aging Research Center, Chang Gung University, Taoyuan, Taiwan and ${ }^{7}$ Neuroscience Research Center, Chang Gung Memorial Hospital, Linkou, Taiwan

Correspondence: G-J. Huang (gjh30@mail.cgu.edu.tw)

These authors contributed equally: Simon Chang, Philane Bok.
}

Received: 9 January 2018 Revised: 5 May 2018 Accepted: 6 May 2018

Published online: 11 May 2018 
C and humidity of $60-70 \%$. Animals had ad libitum access to food and water. All protocols in this study were reviewed and approved by the Institutional Animal Care and Use Committee at Chang Gung University, Taiwan (IACUC: CGU15-063).

Crh (Crh::Cre; Ai9) and Crhr1 (Crhr1::Cre; Ai9) reporter mice for colocalization studies were generated by breeding respective Cre drivers [16] to Ai9 (R26 ${ }^{\text {CAG:IoxP-STOP-loxP-tdTomato, stock no: 007905) }}$ mice purchased from the Jackson Laboratory. Animal experiments were conducted in accordance with the Guide for the Care and Use of Laboratory Animals of the Government of Bavaria, Germany.

\section{Behavioral testing}

To assess anxiety behavior, mice were subjected to the open field, elevated-O-maze, light-dark box, and novelty-suppressed feeding tests. The movement of animals was tracked using Ethovision software. There was a one day rest between each test. Mice were removed randomly to a transferring cage for testing, and stayed in a separated cage with bedding, food and water before being transferred back to the home cage. All mazes are made of acrylic; there is no bedding in the maze during the test.

Open field test. Animals were allowed to freely move for $5 \mathrm{~min}$ in an arena (radius $=45 \mathrm{~cm}$, inner circle with radius $=30 \mathrm{~cm}$ ).

Light dark box. At the start of the test, mice were put in the covered dark (black) box and permitted to move freely between the dark and the coverless light (white) box for 5 min.

Elevated-O-maze. The apparatus consisted of two open arms and two closed arms of equal size. The maze was a circle (radius $=55$ $\mathrm{cm}$ ) elevated $60 \mathrm{~cm}$ above the floor. The closed arms featured a $15 \mathrm{~cm}$ wall. Mice were placed in the elevated-O-maze for $5 \mathrm{~min}$ under dim light. The time spent in the open arms and the number of open arms entries were measured.

Novelty-suppressed feeding. Food was removed from the home cage for $24 \mathrm{~h}$ before testing. For testing, mice were placed in a brightly lit standard rat cage featuring food pellets placed in the four corners. The latency to first approach the food, and the time spent eating in the 7-min test period was recorded manually using a stopwatch.

Home cage food consumption test. To measure chronic food consumption, food pellets were removed for $6 \mathrm{~h}$ prior to being replaced. Food intake was then measured for $1 \mathrm{~h}$. The test was conducted during the active period of mice.

Immunohistochemistry and quantification

Dissected brains were fixed in 4\% paraformaldehyde overnight, following dehydration in $25 \%$ sucrose in phosphate-buffered saline (PBS). All sections for NPTX2, DCX (doublecortin), Ki67, BrdU (5-bromo-2'-deoxyuridine), and c-Fos staining were sliced at a thickness of $40 \mu \mathrm{m}$. Brain sections were mounted on SuperFrost Plus slides (Thermo) and dried overnight. Slides were then incubated in $0.01 \mathrm{M}$ citric acid buffer for $15 \mathrm{~min}$ at $95^{\circ} \mathrm{C}, 3 \%$ $\mathrm{H}_{2} \mathrm{O}_{2}$ for 10 min, rinsed in PBS, and incubated overnight at room temperature in NPTX2 antibody (1:1000, Proteintech), DCX antibody (1:250, Santa Cruz), Ki67 antibody (1:1000, Vector Lab), BrdU antibody (1:250, Accurate), or c-Fos antibody (1:1000, Santa Cruz). Subsequently, we used a standard lgG ABC kit (Vector Lab) procedure according to the manufacturer's instructions and incubated the slide for 5-10 min with a Sigma DAB tablet. Sections were then counterstained with cresyl violet and mounted in DPX.

For BrdU/NeuN double labeling, sections were incubated in $2 \mathrm{M}$ $\mathrm{HCl}$ for 30 min at $37^{\circ} \mathrm{C}$, neutralized in boric acid (Sigma) for $15 \mathrm{~min}$ $(\mathrm{pH} 8.5)$ and washed three times in PBS before incubation with
BrdU antibodies (1:250, Accurate) and NeuN (1:400 Millipore). Following three washes in PBS (5 min each), sections were incubated with the fluorescent secondary antibody $(1: 250$, Alex Fluor 488 and Texas Red, Invitrogen) for $2 \mathrm{~h}$ in $0.3 \%$ Triton/PBS with $2 \%$ of goat serum.

For NPTX2, NeuN, and GFAP immunofluorescence, sections were mounted on SuperFrost Plus slides and dried overnight. Then, slides were incubated in $0.01 \mathrm{M}$ citric acid buffer for $15 \mathrm{~min}$ at $95^{\circ} \mathrm{C}$, rinsed in PBS and incubated overnight at room temperature in rabbit anti-NPTX2 antibody (1:1000, Proteintech), mouse anti-NeuN antibody (1:400, Millipore), mouse anti-GFAP antibody (1:1000, Sigma). Slides were then incubated with suitable fluorescence secondary antibody (1:250 Alexa 488 and Alexa 568, Invitrogen) and then washed with PBS. Slides were cover slipped with mounting medium (Fluoromount-G, SouthernBiotech) and left to either air dry or cooled to $-20^{\circ} \mathrm{C}$ for photo shooting.

For quantification, all slides were randomized and coded before quantitative analysis. Slides (half brain) were examined under a $20 \times$ objective. DCX, Ki67, and BrdU labeled cells were counted on every eighth section through the entire rostrocaudal extent of the granule cell layer (six sections per animal). The number of cells counted was then multiplied by sixteen to obtain an estimate of the total number of DCX, Ki67, and BrdU-positive cells in the dentate gyrus. For c-Fos and NPTX2 labeled cells, we counted cells on every eighth section through the entire rostrocaudal extent of the dentate gyrus, CA1 and CA3 areas (six sections per animal). The raw data was then modified as detailed above.

\section{Double in situ hybridization}

Mice (10-week old) were sacrificed by an overdose of isoflurane. Brains were removed and shock-frozen on dry ice. Frozen brains were cut into 20- $\mu \mathrm{m}$ thick sections and mounted on SuperFrost Plus slides. All sections were processed for double in situ hybridization using a protocol described in a previous study [17]. The following riboprobes were used: Gad67: 984-1940 bp of NM_008077; Gad65: 753-1600 bp of NM_008078; Vglut1 (Slc17a7): $1716-2332 \mathrm{bp}$ of NM_010484; Nptx2: 1668-2384 bp of NM_008078 and Fkbp5: 1481-2328 bp of NM_010220. These were generated from PCRII TOPO vectors containing the CDNA insert. Molecular cloning was done per the manufacturer's instructions (TOPO ${ }^{\circledR}$ TA Cloning ${ }^{\circledR}$ Kit, Cat.450640, Thermo Fisher). Specific riboprobes were generated by PCR from T7 and SP6 primers using plasmids containing the above-mentioned cDNAs as templates. Antisense and sense CRNA probes were synthesized and labeled with $S^{35}$ UTP or dioxygenin (DIG) by in vitro transcription from 1.5 $\mu \mathrm{g}$ of respective PCR product used as templates. For DIG detection, anti-DIG-POD (Fab) antibody was used at a concentration of $1 / 400$. Tiramide-biotin signal amplification (TSA) was performed using the NEL700A Kit as per the manufacturer's instructions. Hybridized slides were dipped in autoradiographic emulsion (type NTB2) and developed after 1 week.

\section{Corticosterone assay}

Blood samples were collected 0, 30, and $90 \mathrm{~min}$ after stress via facial vein puncture. Plasma was separated from whole blood by centrifugation and stored at $-80^{\circ} \mathrm{C}$ until used. For analysis, plasma was diluted $1: 30$ in buffer and measured using the Corticosterone EIA kit (Enzo Life Sciences) as per the manufacturer's instructions.

Viral vector preparation and injections

For the Nptx2 knockdown experiment, an AAV9-Cre vector (AV-9PV2004) and AAV9-eGFP control vector (AV-9-PV0101) were purchased from vector core, University of Pennsylvania. For Nptx2 overexpression and control vectors, DNA fragments that encoded NPTX2 or eGFP were created by PCR and subcloned into the Notl site of a AAV9 virus construct. Recombinant AAV9 vectors were 
a

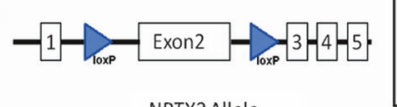

NPTX2 Allele
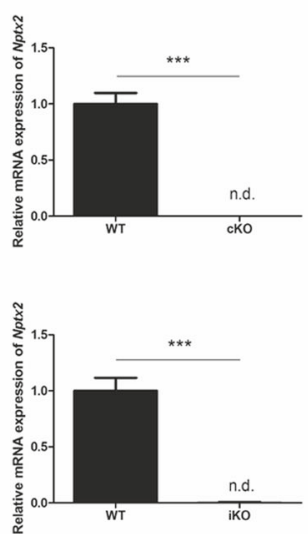

C
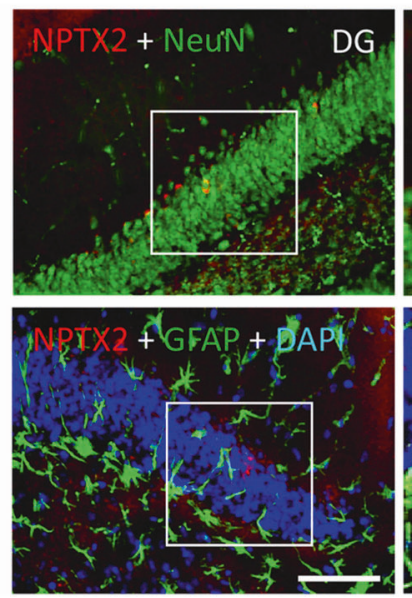

WT: $N p t \times 2^{\mathrm{f} / \mathrm{f}}$

cKO: Nptx $2^{f / f}-$ Sox1::Cre

WT: Nptx $2^{f / f}$

iKO: Nptx $2^{f / f}-$ CAG::CreER ${ }^{T 2}$

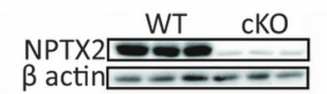

$\beta$ actin $-\ldots$
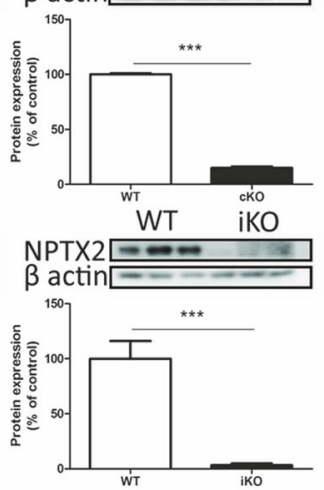

b

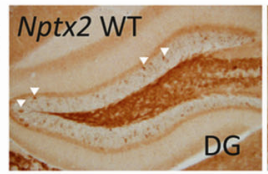

DG
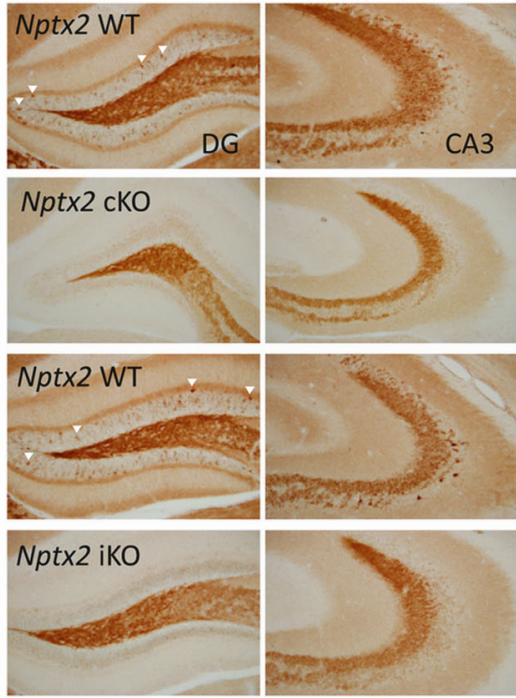

CA3
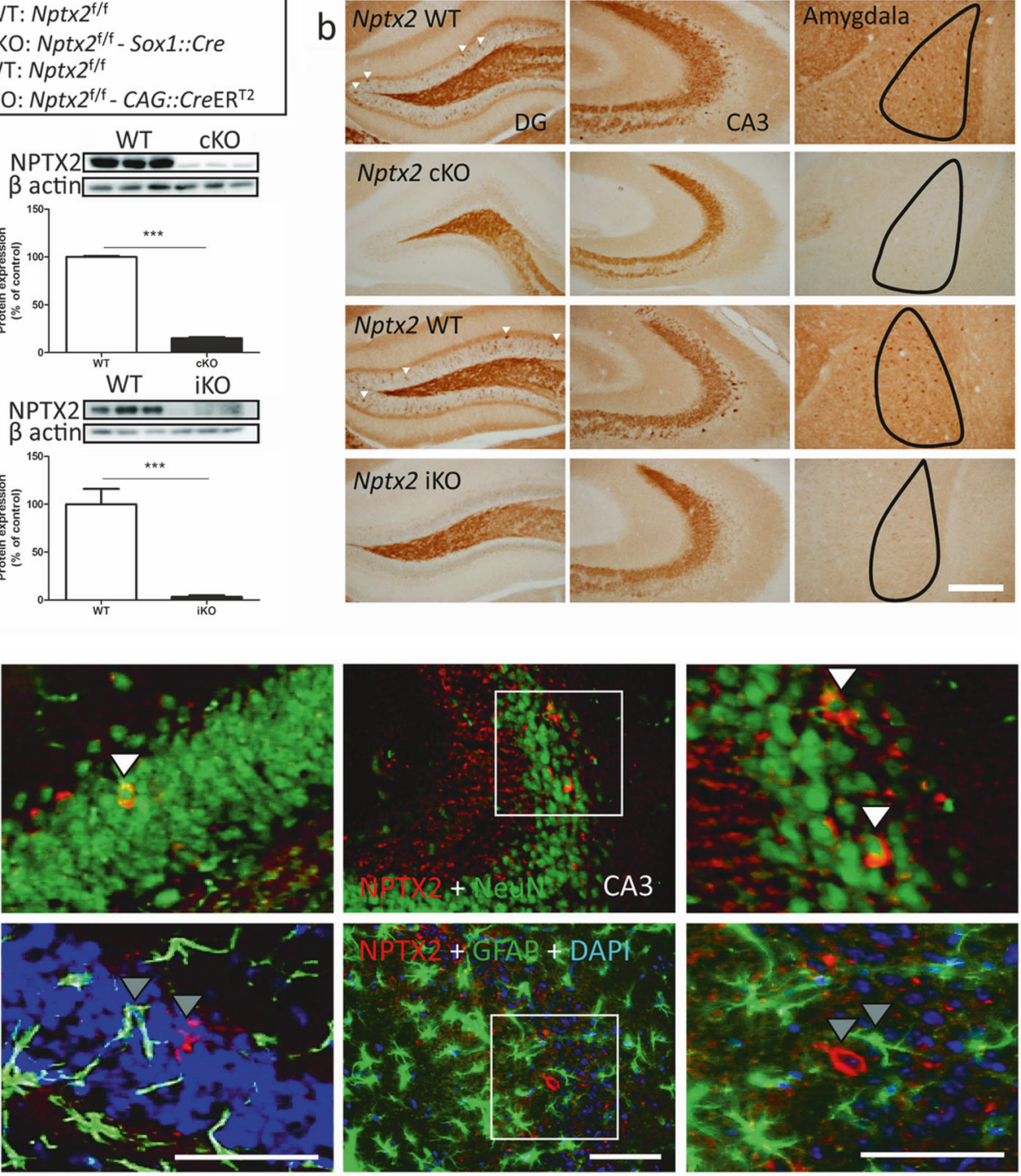

Fig. 1 Generation of Nptx2 conditional knockout mice. a Nptx2 floxed mice were crossed with Sox $1::$ Cre mice to knockout Nptx2 specifically in the brain from early development or with CAG::CreER ${ }^{T 2}$ to knockout Nptx2 in adulthood. We confirmed the high knockout efficiency of Nptx2 in the hippocampus at mRNA $(n=8)$ and protein levels $(n=6)$ for both models. $\mathbf{b}$ Immunohistochemistry confirmed the absence of NPTX2positive cells in both the hippocampus and amygdala. c NPTX2 co-localized with NeuN (a marker for neurons), but not GFAP (a marker for glia). Gray arrowheads indicate no colocalization. White arrowheads indicate colocalization of two markers. Scale bar represents $200 \mu \mathrm{m}$. Values represent mean \pm SEM. ${ }^{* * *} p<0.001$

produced by a standard triple-plasmid transfection method and purified by two rounds of $\mathrm{CsCl}$ centrifugation. The physical vector titers of AAVs were quantified using a real-time PCR method to measure the number of packaged vector genomes.

For the viral vector injections, surgery was performed under anesthesia. Mice received bilateral injections of viral vector into the hippocampus in a volume of $1.5 \mu \mathrm{l}$ each injection side $(4 \times$ $10^{12} \mathrm{GC} / \mathrm{ml}$ for AAV9-Cre and control-eGFP viral vector; $5 \times 10^{13}$ $\mathrm{GC} / \mathrm{ml}$ for AAV9-Nptx2 and control-eGFP) under stereotaxic guidance. For hippocampal injections, there were four injection sites in each mouse (dorsal hippocampus: AP $-2.2 \mathrm{~mm}, \mathrm{ML} \pm 2.0$ $\mathrm{mm}$ from bregma and DV $-1.8 \mathrm{~mm}$ from dura; ventral hippocampus: $\mathrm{AP}-3.0 \mathrm{~mm}, \mathrm{ML} \pm 3.0$ and $\mathrm{DV}-3.3 \mathrm{~mm}$ ). For amygdala injections, there were two injection sites in each mouse (AP -1.75 $\mathrm{mm}, \mathrm{ML} \pm 3.1, \mathrm{DV}-4.0 \mathrm{~mm})$.

\section{Statistical analysis}

The mean \pm SEM was determined for each group. Statistical analysis was performed using Graphpad Prism software. Data were analyzed via an analysis of variance (ANOVA) or $t$-test, as appropriate. Differences were considered significant when $p<0.05$.

Supplementary methods

For the detailed description of RNA seq-based gene expression analysis, mRNA quantification, Western blot analysis, see Supplementary Methods and Materials.

\section{RESULTS}

Generation of nervous system-specific Nptx2 KO mice

To study the role of NPTX2 in emotion related behaviors, we confirmed the expression of Nptx2 in the murine brain using ISH (Supplementary Fig. S1a-e). To develop nervous system-specific Nptx2 conditional KO mice (cKO), we generated loxP-flanked Nptx2 $\left(N p t \times 2^{\mathrm{f} / \mathrm{f}}\right)$ mice. We crossed Nptx2 floxed mice with Sox $1::$ Cre mice, which specifically express Cre throughout the neural tube from E11 [18]. These tissue specific CKO mice permitted an investigation into the functions of Nptx2 in the brain. First, we demonstrated that Nptx2 expression is dramatically decreased in the 


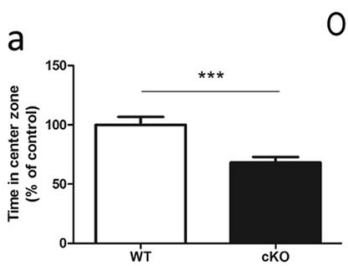

Open Field

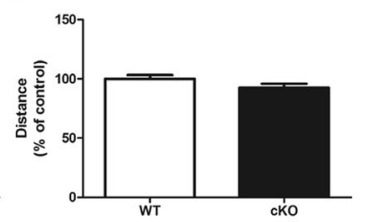

Elevated-O-maze
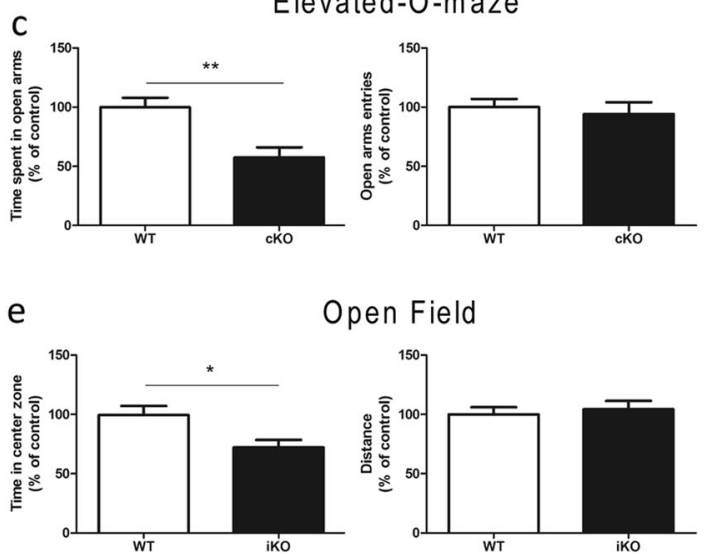

Open Field

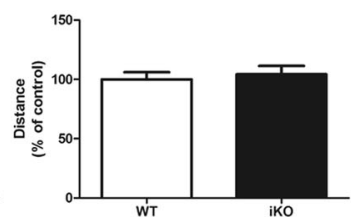

g

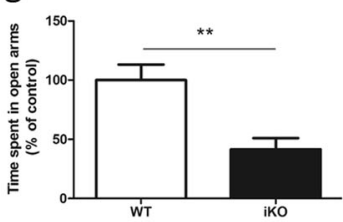

Elevated-O-maze

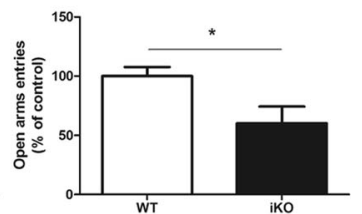

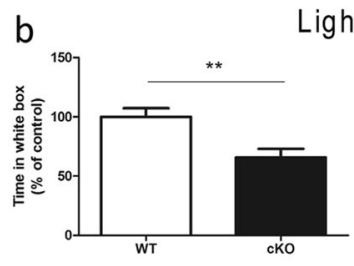

Light Dark box

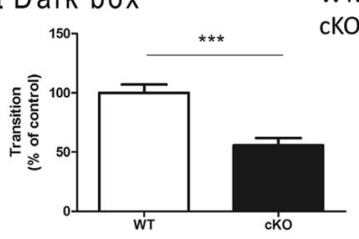

d
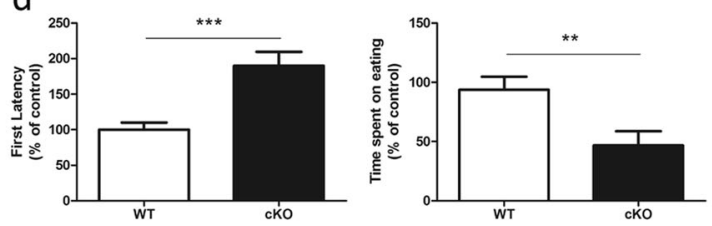

f

Light Dark box
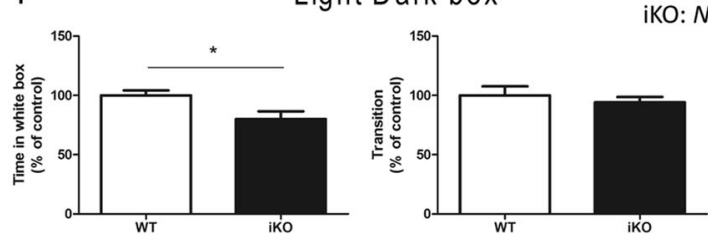

WT: $N p t \times 2^{\mathrm{t} / \mathrm{f}}$

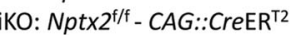

WT: Nptx $2^{\mathrm{f} / \mathrm{f}}$ cKO: Nptx2 $2^{f / f}-S o x 1:: C r e$

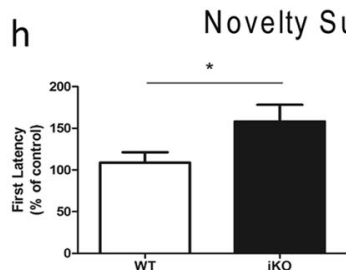
.

Fig. 2 Nptx2 knockout mice show increased anxiety. a-d Mice with brain-specific Nptx2 KO (Sox1::Cre) (WT, $n=24 ;$ cKO, $n=21)$ and e-h tamoxifen-induced KO (CAG::CreER ${ }^{T 2}$ ) (WT, $n=14$; iKO, $\left.n=11\right)$ showed increased anxiety behavior. a, e In the open field, KO mice spent less time in the central zone of the open field without a significant difference in distance traveled. $\mathbf{b}, \mathbf{f}$ In the light-dark box, WT mice stayed longer in the white box and made more transitions between the two boxes. $\mathbf{c}, \mathbf{g}$ In the elevated-O-maze, KO mice spent less time in the open arms and made a similar number of arm entries compared with WT. $\mathbf{d}, \mathbf{h}$ In the novelty-suppressed feeding test, WT mice approached food pellets faster and spent more time eating than KO mice. Values represent mean \pm SEM. ${ }^{*} p<0.05,{ }^{* *} p<0.01,{ }^{* * *} p<0.001$

hippocampus by measuring mRNA via quantitative polymerase chain reaction (qPCR) $(p<0.0001, t=10.14, n=8)$ and protein levels via western blot $(p<0.0001, t=50.81, n=6)$ (Fig. 1a). Immunohistochemistry (IHC) showed only a few NPTX2-positive cells remained in CKO mice (Fig. 1b). On the IHC stains, we notice that some mossy fiber staining can be observed in Nptx2 KO mice. Since the western and qPCR data demonstrates very low levels of this gene and protein in $\mathrm{KO}$, this remaining staining should be a result of nonspecific staining. Next, by using $\mathrm{IHC}$, we demonstrated that NPTX2 co-localizes with the neuronal marker NeuN, but not the glial marker GFAP (Fig. 1c). We then showed Nptx2 mRNA co-localizes with Vglut1 in the dentate gyrus (DG) and CA3 using double ISH. However, we detected minimal colocalization with Gad65/Gad67. This suggests that Nptx2 is expressed primarily in excitatory neurons of the hippocampus (Supplementary Fig. S1f-i).

Increased anxiety responses in Nptx2 cKO mice

To investigate the relationship between Nptx2 and anxiety, we subjected mice to a battery of behavioral tests (WT, $n=24$; cKO, $n$ $=21$ ). This battery included the open field test, light-dark box, elevated-O-maze, and novelty-suppressed feeding. Overall, our results showed that Nptx2 deletion robustly increased anxiety-like behaviors. In the open field, cKO mice spent less time in the center zone $\left(p<0.001, t_{(43)}=3.84\right)$ with no significant difference in the total distance traveled $\left(p=0.11, t_{(43)}=1.65\right) \quad$ (Fig. 2a). In the light-dark box, cKO mice spent less time in the white box $\left(p=0.001, t_{(43)}=3.33\right)$ and made fewer transitions between the two compartments $\left(p<0.0001, t_{(43)}=4.64\right)$ (Fig. 2b). In the elevated-O-maze, we found that CKO mice spent less time in the open arms $\left(p=0.001, t_{(43)}=3.65\right)$, but they did not exhibit a significant difference in the number of open arm entries $(p=0.63$, $t_{(43)}=0.49$ ) (Fig. 2c). Our results indicate cKO mice express higher levels of anxiety than WT mice. To further confirm this conclusion, we conducted an activity independent anxiety test, the noveltysuppressed feeding test. This test revealed that CKO mice exhibit a longer latency to approach food pellets $\left(p=0.0002, t_{(43)}=4.18\right)$ and spend less time eating in a new environment $\left(p=0.008, t_{(43)}\right.$ $=2.87$ ) (Fig. 2d). WT and cKO mice had comparable home cage food consumption $\left(p=0.37, t_{(43)}=1\right)$. Altogether, these results further demonstrate that Nptx2 plays an important role in mediating anxiety.

Altered hippocampal progenitor cell proliferation in Nptx2 cKO mice

Nptx2 is expressed in the dentate gyrus and is upregulated following BDNF infusion, antidepressant treatment, and exercise $[1,12]$. Since these factors play a significant role in neurogenesis $[19,20]$, we asked whether Nptx2 contributes to the regulation of adult hippocampal neurogenesis. To investigate this, we injected mice with 5-bromo-2'-deoxyuridine (BrdU) (200 mg/kg, i.p.) to label newly born cells. Mice were killed 28 days after this BrdU injection to evaluate cell survival. We also assessed proliferation of neuronal progenitors by staining for doublecortin (DCX), a marker 


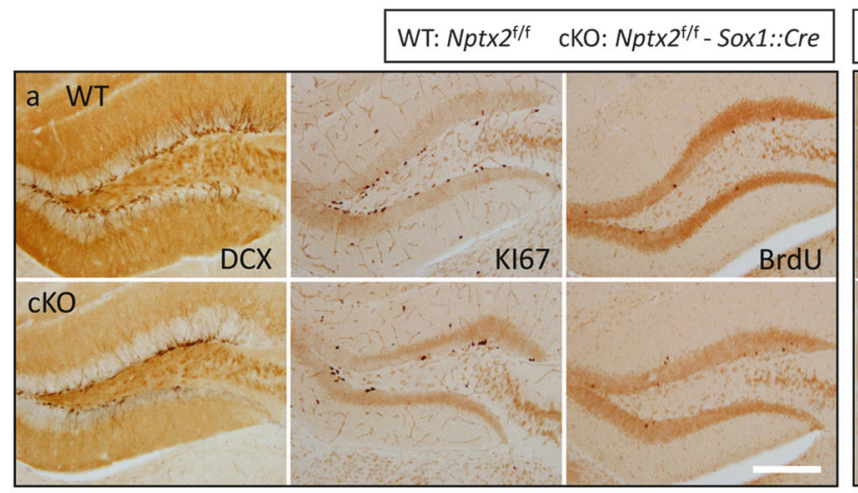

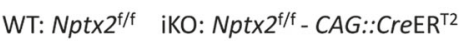
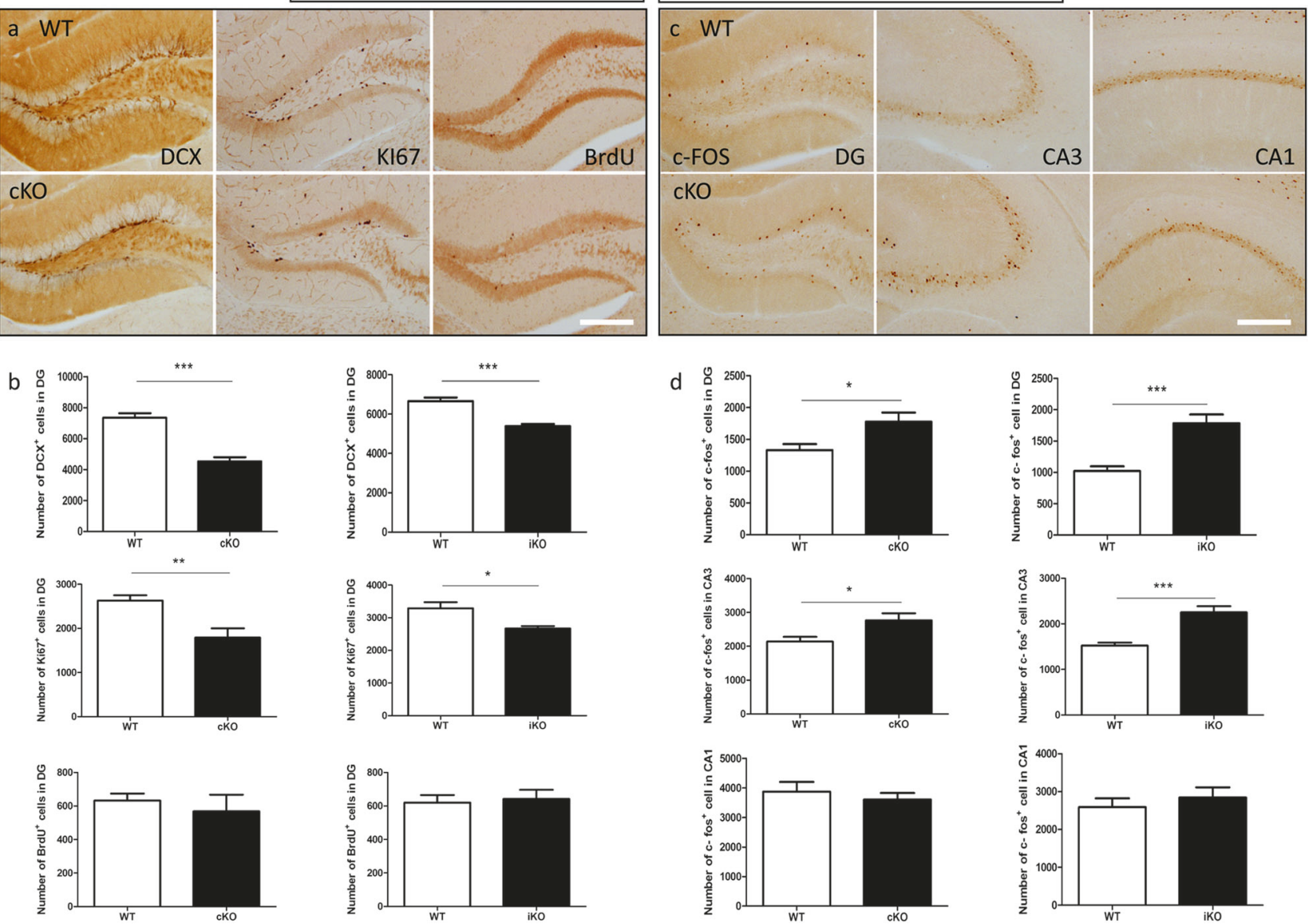

Fig. 3 Nptx2 knockout mice exhibit aberrant hippocampal cell proliferation and increased stress-induced neuronal activity. a Images of neurogenesis markers DCX, Ki67, and BrdU in the dentate gyrus of the hippocampus. b There were significantly reduced DCX and Ki67 labeled cell counts in the CKO hippocampus, but there were no significant differences in BrdU-positive cell numbers (left, $n=8$ ). Inducible Nptx2 KO had a similar decrease in cell proliferation (right, WT, $n=14$; iKO, $n=11$ ). c, d Images and quantification of c-Fos in the hippocampus DG, CA1, and CA3. Brain-specific KO (left, $n=8$ ) and inducible KO mice (right, WT, $n=14$; iKO, $n=11$ ) have higher levels of c-Fos-positive cells in DG and CA3, but not in CA1 after acute restraint stress. Scale bar represents $200 \mu \mathrm{m}$. Values represent mean $\pm{ }^{\mathrm{SEM}}{ }^{*} p<0.05,{ }^{* *} p<0.01,{ }^{* * *} p<0.001$

for immature neurons, and Ki67, a marker for cell proliferation. We demonstrated that cell proliferation and the number of immature neurons is dramatically decreased in Nptx2 cKO mice $(n=8)$ as measured by $\operatorname{Ki67}\left(p=0.002, t_{(14)}=3.75\right)$ and DCX $(p<0.0001$, $\left.t_{(14)}=6.98\right)$ staining. However, there was no significant difference in BrdU labeled cell counts ( $p=0.5$ ) (Fig. 3a, b left). Approximately $65 \%$ of the BrdU-positive cells were also positive for the neuronal marker, NeuN; however, there were no differences in the percentage of BrdU/NeuN co-labeling between the two groups $(n=6, p=0.58, t=0.68)$. These data reveal that despite the reduction of proliferating cells in the DG in CKO mice, neurogenesis is maintained, presumably due to a higher survival rate. This may reflect a compensation effect regulated by other factors to maintain adequate overall neurogenesis. Overall, these findings suggest that mice without Nptx2 exhibit decreased progenitor cell proliferation but not altered neurogenesis.

Altered stress-induced neuronal activity in Nptx2 cKO mice Altered plasma cortisol and stress-induced dysfunction through the hypothalamic-pituitary-adrenal (HPA) axis are important features in anxiety disorders [21]. To further investigate the role of Nptx 2 in response to stress, we measured plasma corticosterone levels at basal conditions and after 30 and 90 min after 30 min restraint stress. We found that corticosterone levels are higher in
Nptx2 cKO mice $(n=7)$ at the 30-min time point $\left(p=0.007, t_{(12)}=\right.$ $3.23)$, but there were no significant differences at either the basal $\left(p=0.24, t_{(12)}=1.24\right)$ nor 90 -min time point $\left(p=0.19, t_{(12)}=1.36\right)$ (Supplementary Fig. S2a). These results demonstrate that Nptx2 CKO mice exhibit a greater HPA response to stress.

We then predicted that our observed differences in stress responsivity would manifest in concomitant neural activity measures in the hippocampus. c-Fos, an immediate early gene, is commonly used as a neuronal activity marker following a stimulus [22]. To characterize neuronal activity in WT and cKO hippocampi in response to stress $(n=8)$, we measured neuronal activation by quantifying the number of c-Fos-positive cells $90 \mathrm{~min}$ after $30 \mathrm{~min}$ of restraint stress in both WT and CKO mice. Interestingly, cKO mice showed more c-Fos-positive cells in the DG $\left(p=0.018, t_{(14)}=2.68\right)$ and CA3 $\left(p=0.021, t_{(14)}=2.61\right)$, but not in CA1 $\left(p=0.5, t_{(14)}=\right.$ 0.68 ) and amygdala $\left(p=0.18, t_{(14)}=1.41\right)$ (Fig. 3c, d left, Supplementary Fig. S3), suggesting Nptx2 null mice display increased hippocampal neuronal activity in response to stress.

The increased anxiety responses and altered hippocampal cell proliferation in Nptx2 KO mice are not due to abnormal brain development

To determine whether the abnormal behaviors we observed in $N p t \times 2$ CKO mice was a consequence of Nptx2 inactivation during 
early embryonic development, we crossed Nptx2 floxed mice with $C A G:: C_{r e E R}{ }^{12}$ mice to induce loss of Nptx2 specifically in adulthood upon tamoxifen administration (WT: Nptx $2^{\mathrm{f} / \mathrm{f}}$; iKO: $\left.N p t \times 2^{\mathrm{f} / \mathrm{f}}-\mathrm{CAG}:: \mathrm{CreER}^{\mathrm{T} 2}\right)$. Fourteen days after three daily injections of tamoxifen $(100 \mathrm{mg} / \mathrm{kg}$, i.p.), we assayed all mice using our behavioral battery described above (WT, $n=14$; iKO, $n=11$ ). In the open field, Nptx2 iKO mice spent less time in the center zone $\left(p=0.02, t_{(23)}=2.47\right)$ with no significant difference in the total distance traveled $\left(p=0.64, t_{(23)}=0.46\right)$ (Fig. 2e). In the light-dark box test, iKO mice spent less time in the light compartment ( $p=$ $0.012, t_{(23)}=2.7$ ) with no significant differences in the number of transitions between the two boxes $\left(p=0.54, t_{(23)}=0.61\right)$ (Fig. $\left.2 f\right)$. In the elevated-O-maze, iKO mice spent less time in the open arms $\left(p=0.004, t_{(23)}=3.5\right)$ and made fewer entries into the open arms ( $\left.p=0.023, t_{(23)}=2.57\right)$ (Fig. 2g). Finally, iKO mice showed a longer latency to approach food pellets $\left(p=0.011, t_{(23)}=2.79\right)$ and spent less time eating ( $\left.p=0.01, t_{(23)}=2.89\right)$ (Fig. $\left.2 \mathrm{~h}\right)$ in the novelty-suppressed feeding test. We also found no significant differences in home cage food consumption between WT and iKO mice $\left(p=0.15, t_{(23)}=2.3\right)$. We assayed both Nptx2 mRNA and protein levels in these mice (qPCR: $p=0.0002, t=13.28, n=8$; Western blot: $p=0.0001, t=4.81, n=6$ ). IHC analysis confirms there were significantly fewer NPTX2-positive cells in the hippocampus and amygdala in Nptx2 iKO after tamoxifen injection (Fig. 1a, b).

We further analyzed hippocampal neurogenesis in these Nptx2 inducible KO mice (WT, $n=14$; iKO, $n=11$ ). We found mice with induced Nptx2 deletion display exhibit fewer DCX $(p<0.0001$, $\left.t_{(23)}=5.39\right)$ and Ki67 $\left(p=0.01, t_{(23)}=2.8\right)$ positive cells in the DG, but no significant differences in BrdU-positive cells $\left(p=0.76, t_{(23)}=\right.$ 0.31) (Fig. 3b right). However, induced Nptx2 deletion yielded no significant differences in the plasma corticosterone level at any time after restraint stress (WT, $n=14$; iKO, $n=11$ ) (Supplementary Fig. S4b). Inducible KO mice exhibited greater c-Fos-positive cells in the DG $\left(p<0.0001, t_{(23)}=5.06\right)$ and CA3 $\left(p<0.0001, t_{(23)}=5.21\right)$, but not CA1 $\left(p=0.5, t_{(23)}=0.69\right)$ and amygdala $\left(p=0.085, t_{(23)}=\right.$ 1.81), 90 min following $30 \mathrm{~min}$ of restraint stress (Fig. 3d right and Supplementary Fig. S3). These data suggest that the altered anxiety and progenitor cell proliferation can occur as a consequence of Nptx2 inactivation in adulthood, and not only because of developmental defects. Furthermore, we demonstrate that these changes in anxiety when the gene is deleted in adulthood are not due to corticosterone overproduction after stress.

Hippocampus-specific knockout of Nptx2 is sufficient to increase anxiety responses

Since chronic antidepressant treatment or exercise upregulates Nptx2 expression in the hippocampus [1, 13] and Nptx2-deficient mice display more c-Fos-positive cells in the DG and CA3 regions but not the amygdala after acute stress, we hypothesized that hippocampal Nptx2 modulates anxiety behavior. To answer this, we injected either AAV-Cre or AAV-eGFP viral vectors into both the dorsal and ventral hippocampus in $N p t x 2^{\mathrm{f} / \mathrm{f}}$ mice $(n=10)$. Fourteen days after surgery, we assayed mice in our behavioral battery: open field, light-dark box, elevated-O-maze, and noveltysuppressed feeding tests. Our results show that $N p t \times 2^{\mathrm{f} / \mathrm{f}}$ mice injected with AAV-Cre (Nptx $2^{\mathrm{f} / \mathrm{f}}$-Cre) showed increased anxiety compared to the injected control AAV-eGFP group (open field: distance, $p=0.17, t_{(18)}=1.4$; time in center zone, $p=0.04, t_{(18)}=$ 2.21; light-dark box: time in white box, $p<0.0001, t_{(18)}=5.06$; transitions, $p=0.03, t_{(18)}=2.24$; elevated-O-maze: time in open arms, $p=0.005, t_{(18)}=3.31$; open arms entries, $p=0.003, t_{(18)}=$ 3.45; novelty-suppressed feeding: first latency, $p=0.03, t_{(18)}=$ 2.27; time spent on eating, $p=0.01, t_{(18)}=2.77$; food consumption, $p=0.32, t_{(18)}=1.3$ ) (Fig. 4a). These results are consistent with our data from CKO and iKO animal models.

Next, we subjected these mice to acute restraint stress and collected plasma after 30 minutes to assay corticosterone level.
We found no significant differences in stress-induced plasma corticosterone levels between the two groups $(p=0.73, t=0.35$, $n=8$ ) (Supplementary Fig. S2c). These results suggest that hippocampal Nptx2 does not alter stress-induced corticosterone release. After harvesting the hippocampal tissue, we confirmed local inactivation of hippocampal Nptx2 in this cohort of mice measured by two assays (qPCR, $p=0.0003, t=6.19$; IHC, $p=$ $0.008, t=10.58, n=6$ ) (Fig. $4 \mathrm{~b}-\mathrm{d}$ ).

The amygdala plays a crucial role in fear and anxiety [23]. Since Nptx2 is expressed in amygdala, we examined the contribution of amygdala Nptx2 in anxiety via injecting AAV-Cre or AAV-eGFP viral vectors into the amygdala in $N p t x 2^{f / f}$ mice $(n=8)$. Fourteen days after surgery, we assayed mice in the behavioral battery described above. Our results showed no significant differences between amygdala Nptx2 knockout and controls in the open field test (distance $p=0.49, t_{(14)}=0.7$; time in center zone $p=0.47, t_{(14)}=$ 0.75 ), light-dark box (time in white box $p=0.63, t_{(14)}=0.5$; transition $p=0.21, t_{(14)}=1.3$ ), elevated-O-maze (time in open arms $p=0.46, t_{(14)}=0.77$; open arms entries $p=0.45, t_{(14)}=0.77$ ). For the novelty-suppressed feeding test, the amygdala Nptx2 knockout mice spent more time eating, but we found no significant differences in first latency and food consumption (first latency $p=0.65, t_{(14)}=0.47$; time spent eating $p=0.0001, t(14)=$ 5.2; food consumption $p=0.52, t_{(14)}=0.65$ ) (Supplementary Fig. S4d-g). We confirmed local inactivation of amygdala Nptx2 in these mice as measured by $\operatorname{IHC}\left(p<0.0001, t_{(14)}=7.25\right)$ (Supplementary Figure S4a-c). Altogether, these data suggest that Nptx2 expression in the hippocampus, but not the amygdala, plays a critical role in anxiety-related behaviors.

Nptx2 modulates hippocampal corticotropin-releasing hormone and glucocorticoid-related pathways

To determine the mechanism through which Nptx2 alters anxiety, we assessed differences in hippocampal gene expression between brain-specific Nptx2 cKO and WT mice by RNA-sequencing (RNAseq; $n=3$ ). We found 397 genes with differential expression. Of these, we selected 53 genes $(p<0.05$, fold change $>1.5$ or $<$ -1.5 ), excluding genes with low expression (expression value $>$ 0.1) (Supplementary Fig. S5). Among these candidate genes, we found that corticotropin-releasing hormone $(\mathrm{Crh})$ was strongly upregulated $(p=0.00038$, fold change $=1.69$ ) .

Hippocampal Crh, mineralcorticoid receptor (MR), glucocorticoid receptor (GR), and GR downstream genes play important roles in anxiety [24-27]. We examined whether Nptx2 alters the expression of $\mathrm{Crh}$ and glucocorticoid-related genes by assaying the differences in hippocampal gene expression between Nptx2 WT and cKO mice $(n=8)$. First, we confirmed increased expression of hippocampal Crh in Nptx2 cKO mice $\left(p=0.001, t_{(14)}=3.82\right)$ (Fig. 5a). Using IHC, we demonstrated that NPTX2 co-localizes with corticotropin-releasing hormone receptor 1 (CRHR1), but not CRH containing neurons (Supplementary Fig. S1I-o). Since Nptx2 alters the expression of hippocampal Crh, we investigated the expression of glucocorticoid signaling related genes in the hippocampus, including $\mathrm{Nr} 3 \mathrm{Cl}$ (GR), Nr3c2 (MR), Crhr1, Sgk1, Fkbp5, and Gilz. We found that Nptx2 cKO mice exhibit higher levels of MR ( $p=0.0018$, $\left.t_{(14)}=3.82\right)$ and Crhr1 $\left(p=0.005, t_{(14)}=3.36\right)$, but we found no significant differences in GR downstream genes (Fig. 5a). For tamoxifen-induced Nptx2 deletion mice $(n=8)$, we confirmed upregulated $\mathrm{Cr} h$ in Nptx2 iKO mice $\left(p=0.02, t_{(14)}=2.55\right)$. There were no differences in MR, Crhrl, and GR downstream gene expression (Fig. 5b).

Interactions between genetic predisposition and environment play an important role in the development of psychiatric disorders. To determine whether Nptx2 alters the expression of glucocorticoid signaling related genes after stress, Nptx2 WT and cKO mice $(n=8)$ experienced restraint stress for $30 \mathrm{~min}$ and were sacrificed after $90 \mathrm{~min}$. We detected several gene expression changes in the hippocampus of CKO mice following acute stress, 
a

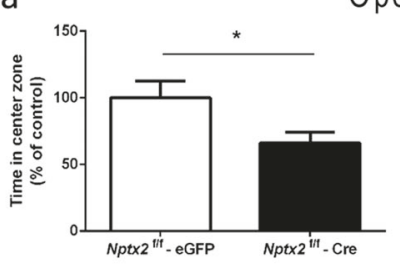

Elevated-O-maze
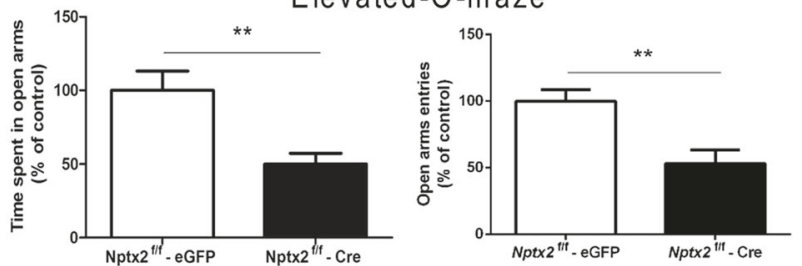

Light Dark box
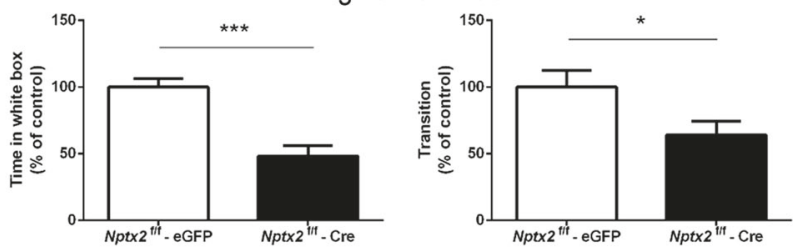

Novelty Suppressed Feeding
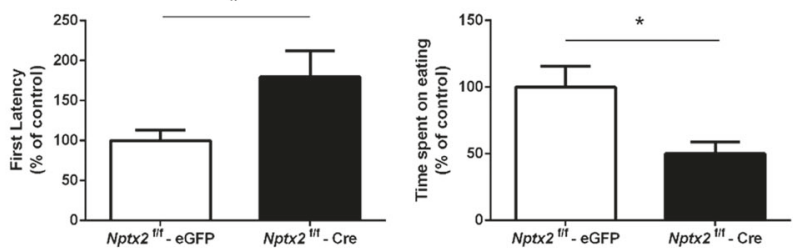

b
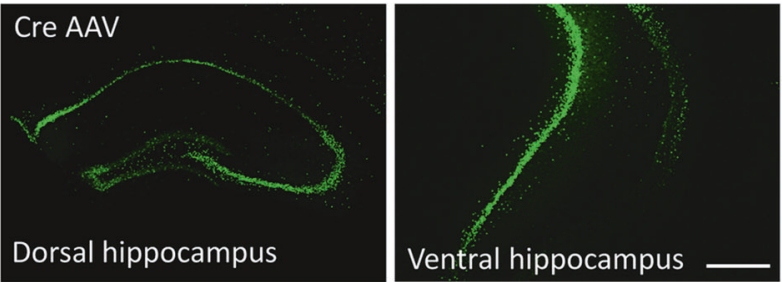

C
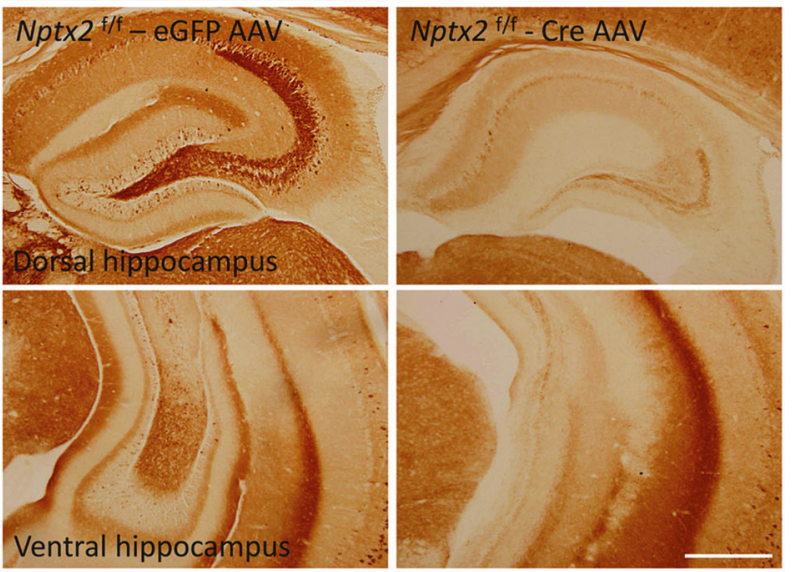

$\mathrm{d} \stackrel{\mathrm{X}}{\mathrm{z}}$
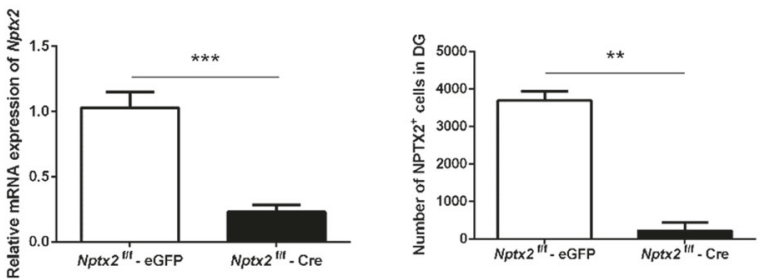

Fig. 4 Hippocampal Nptx2 knockdown by AAV-Cre injection causes increased anxiety. a Mice injected with AAV-Cre virus featured significantly increased anxiety. This is demonstrated by a decrease in time spent in the central zone (open field), less time spent in the open arms and fewer entries to the open arms (elevated-O-maze), spent less time in and made less transition to the white box (light-dark box), exhibited a greater latency to approach food pellets and spent less time eating (novelty-suppressed feeding test) compared to mice with AAV-eGFP virus injection $(n=10)$. b, c Viral vector expression (eGFP) and antibody staining of NPTX2 in the dorsal and ventral hippocampus. Immunohistochemistry confirmed there were no NPTX2-positive cells in the hippocampus. d mRNA expression of Nptx2 and NPTX2positive cell number in the hippocampus $(n=6)$. Scale bar represents $500 \mu \mathrm{m}$. Values represent mean $\pm \mathrm{SEM} .{ }^{*} p<0.05,{ }^{* *} p<0.01,{ }^{* * *} p<0.001$

including upregulation of $\mathrm{Crh}\left(p=0.03, t_{(14)}=2.37\right), \mathrm{GR}(p=0.01$, $\left.t_{(14)}=2.89\right)$ and GR downstream genes, such as Sgk1 $(p=0.01$, $\left.t_{(14)}=2.83\right)$, and Fkbp5 ( $\left.p=0.02, t_{(14)}=2.51\right)$ (Fig. 5c). We also detected similar changes in inducible Nptx2 knockout mice (iKO; Crh $p=0.005, t_{(14)}=3.68$, GR $p=0.01, t_{(14)}=3.01$, Crhr1 $p=0.01$, $\left.t_{(14)}=3.17, F k b p 5 p=0.003, t_{(14)}=3.89\right)$ and Nptx2 floxed mice injected with AAV-Cre into the hippocampus (Nptx $2^{\mathrm{f} / \mathrm{f}}-\mathrm{Cre}, n=6$; Crh $p=0.002, t_{(14)}=4.47$, GR $p=0.004, t_{(14)}=3.68$, Sgk1 $p=$ $0.009, t_{(14)}=3.31, F k b p 5 p=0.0003, t_{(14)}=5.32$, Gilz $p=0.018, t_{(14)}$ $=2.83$ ) (Fig. $5 \mathrm{~d}$, e). These results suggest Nptx2 influences GR, $S g k 1$, and $F k b p 5$ expression during stress.

Since stress increases plasma corticosterone, we wanted to determine whether the expression of these GR-related genes in Nptx2-deficient mice respond differently to elevated plasma corticosterone. We injected both WT and cKO mice $(n=8)$ with a single dose of corticosterone (CORT, $2.5 \mathrm{mg} / \mathrm{kg}$, i.p.) to imitate the adrenal hormone stress response. We detected no significant differences in plasma corticosterone between WT and cKO mice $90 \mathrm{~min}$ after injection (WT $582.7 \pm 44.71 \mathrm{ng} / \mathrm{ml}$; KO $590.6 \pm 56.94$ $\left.\mathrm{ng} / \mathrm{ml}, p=0.91, t_{(14)}=0.1\right)$. Interestingly, cKO mice exhibited higher expression of $\mathrm{Crh}\left(p=0.003, t_{(14)}=3.6\right), \mathrm{GR}\left(p=0.04, t_{(14)}\right.$ $=2.16), \operatorname{Crhr} 1\left(p=0.019, t_{(14)}=2.65\right), \operatorname{Sgk} 1 \quad\left(p=0.04, t_{(14)}=2.26\right)$ and $F k b p 5\left(p=0.0007, t_{(14)}=4.54\right)$ (Fig. $\left.5 f\right)$. These results suggest that NPTX2 regulates sensitivity of GR signaling, rather than corticosterone levels per se.

Overexpression of Nptx2 in the hippocampus reduces stressinduced anxiety

To study the potential of Nptx 2 to be a target for novel treatments for anxiety, we overexpressed Nptx2 in the hippocampus. C57BL/6 mice were separated into two groups $(n=10)$ and injected with either AAV-CB (chicken-beta actin)-Nptx2 viral vector (OE) or with an AAV-CB-eGFP viral vector (Control) into the hippocampus. Fourteen days after surgery, we subjected all mice to behavioral testing. Our results showed no significant differences between $\mathrm{OE}$ mice and controls in the open field test, light-dark box, elevatedO-maze, novelty-suppressed feeding (Supplementary Fig. S6). Hippocampal tissues were harvested and we detected a robust increase in Nptx2 mRNA expression in AAV-CB-Nptx2 viral vector injecting group compared to eGFP control $(p=0.002, t=3.79$. $n$ $=8$ ). These results show that increased hippocampal Nptx2 expression does not alter anxiety.

Because C57BL/ 6 is one of the least anxious inbred mouse strains [28], we tested whether increased Nptx2 can reduce anxiety following stress. Fourteen days after injection of overexpression or control viral vector into the hippocampus (C57BL/6 mice, $n=10$ ), 

WT: Nptx $2^{\mathrm{f} / \mathrm{f}}$
cKO: Nptx2 ${ }^{\mathrm{f} / \mathrm{f}}-$ Sox1::Cre $\quad$ iKO: Nptx $2^{\mathrm{f} / \mathrm{f}}-$ CAG::CreER ${ }^{\mathrm{T} 2}$

a
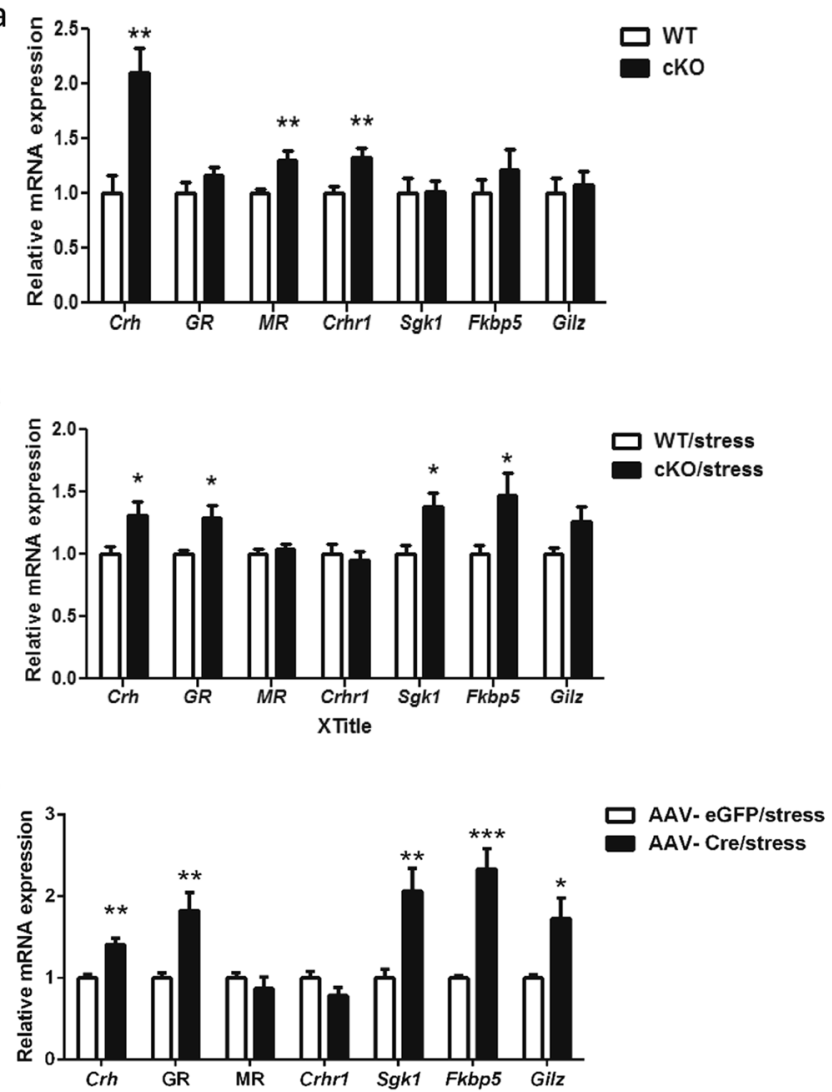

b

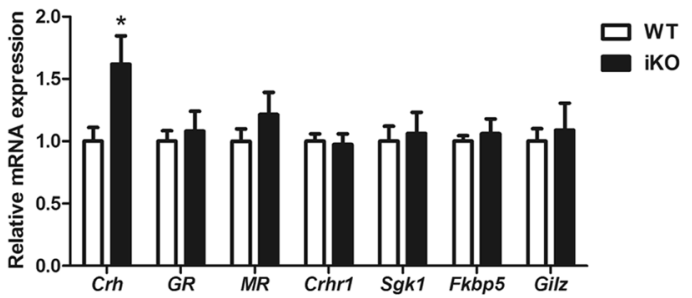

d

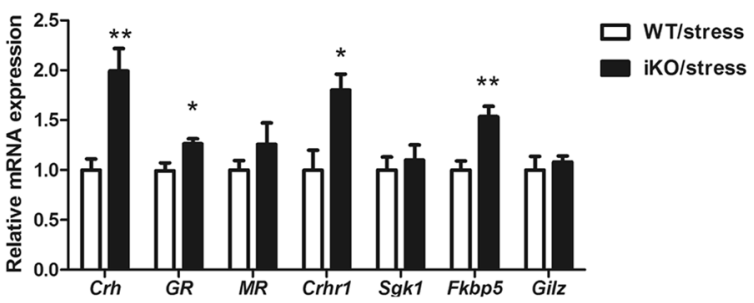

$\left.f \varepsilon^{3}\right] * * \quad \square$ WT/CORT

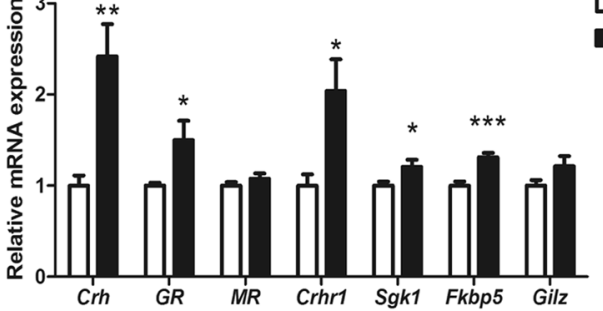

Fig. 5 Nptx2 regulates corticotrophin releasing hormone $(\mathrm{Crh})$ and glucocorticoid pathway gene expression. $\mathbf{a}$, $\mathbf{b}$ Expression of glucocorticoidrelated genes (Crh, GR, MR, Crhr1, Sgk1, Fkbp5, and Gilz) in the hippocampus of Nptx2 KO mice (brain-specific and tamoxifen-induced) $(n=8)$. c, d Gene expression in the hippocampus after $90 \mathrm{~min}$ of initiating $30 \mathrm{~min}$ of restraint stress in brain-specific KO or tamoxifen-induced KO mice $(n=8)$. e Gene expression in the hippocampus of control-eGFP and AAV-Cre injected mice after acute restraint stress $(n=6)$. $f$ Gene expression of the hippocampus of WT and cKO mice 90 minutes after a single corticosterone injection $(2.5 \mathrm{mg} / \mathrm{kg}$, i.p. $n=8)$. Values represent mean \pm SEM. ${ }^{*} p<0.05,{ }^{* *} p<0.01,{ }^{* * *} p<0.001$

all mice were subjected to $3 \mathrm{~h}$ of restraint stress. We initiated behavioral tests following $6 \mathrm{~h}$ of post-stress resting in the home cage. We found that Nptx2 overexpressing mice exhibit less anxious behavior in all four tests compared to eGFP control mice (open field: distance $p=0.47, t_{(18)}=0.73$, time in center zone $p=$ $0.01, t_{(18)}=2.83$; light-dark box: time in white box $p=0.01, t_{(18)}=$ 2.83 , transitions $p=0.22, t_{(18)}=1.25$; elevated-O-maze: time in open arms $p=0.0 .02, t_{(18)}=2.57$; open arms entries $p=0.24, t_{(18)}$ $=1.2$; novelty-suppressed feeding: first latency $p=0.008, t_{(18)}=$ 2.93; time spent on eating $p=0.07, t_{(18)}=1.89$ ) (Fig. 6a-d). This suggests increased $N p t x 2$ is protective against stress-induced anxiety.

We then sacrificed these mice $90 \mathrm{~min}$ following $30 \mathrm{~min}$ of restraint stress and assayed plasma corticosterone. We found no significant differences in plasma corticosterone levels between $\mathrm{OE}$ and control mice ( $p=0.4, t=0.86, n=8$ ) (Supplementary Fig. S2d). After measuring Nptx2 expression levels, we confirmed that mice injected with AAV-CB-Nptx2 viral vector expressed higher levels of Nptx2 mRNA $(p<0.0001, t=6.17 ; n=7)$ (Fig. 6f). We also confirmed that Nptx2 overexpressing mice had more NPTX2positive cells (DG $p=0.0002, t=5.86$; CA3 $p<0.0001, t=18, n=$ 6) (Fig. 6e, g, h). After stress, Nptx2 overexpressing mice exhibited reduced expression of Crhr1, Sgk1, and Fkbp5 (Crhr1 $p=0.02, t=$ 2.68, Sgk1 $p=0.03, t=2.42$, Fkbp5 $p=0.04, t=2.3 ; n=7$ ) (Fig. 6i).
Intriguingly, among these GR-related genes, Fkbp5 expression coincides with Nptx2 present (knockout and overexpression) under stress condition. Double ISH showed that Nptx2 colocalized with Fkbp5 in the DG and CA3 (Supplementary Fig. S1j, k). Overall, these results indicate that increased $N p t x 2$ reverses stress-induced behavioral changes and decreases GR downstream gene expression.

\section{DISCUSSION}

We utilized three gene deletion methods to examine the role of Nptx2 in anxiety: central nervous system-specific, tamoxifeninduced, and hippocampus-specific deletion. Our experiments demonstrate that the effects of Nptx2 deletion on anxiety-like behavior are hippocampus-specific and not developmentally related. In addition, mice with Nptx2 deletion exhibit decreased hippocampal cell proliferation and increased stress-induced neuronal activity. Furthermore, changes in expression of GRrelated genes are consistent with the behavioral results.

Our data show that brain-specific Nptx2 KO mice exhibit increased anxiety. These results are consistent with a recent study that demonstrated double KO mice for Nptx2 and Nptxr exhibit increased anxiety in both the open field and elevated-O-maze [9]. However, in that study, methodological issues may have 
a

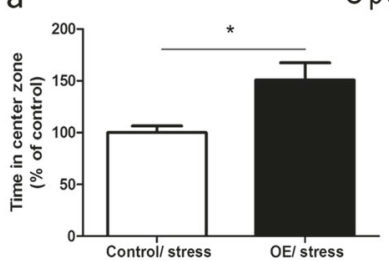

Open Field

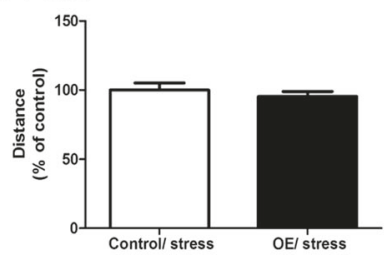

b

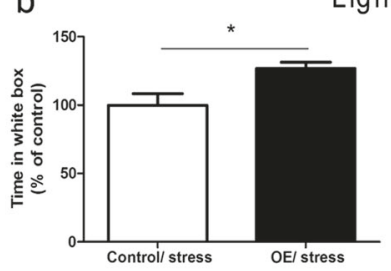

Light Dark box

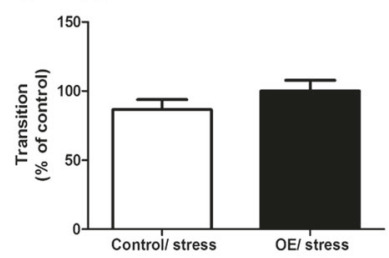

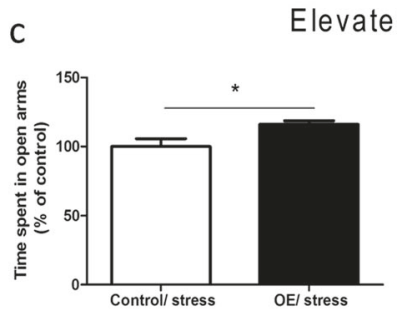
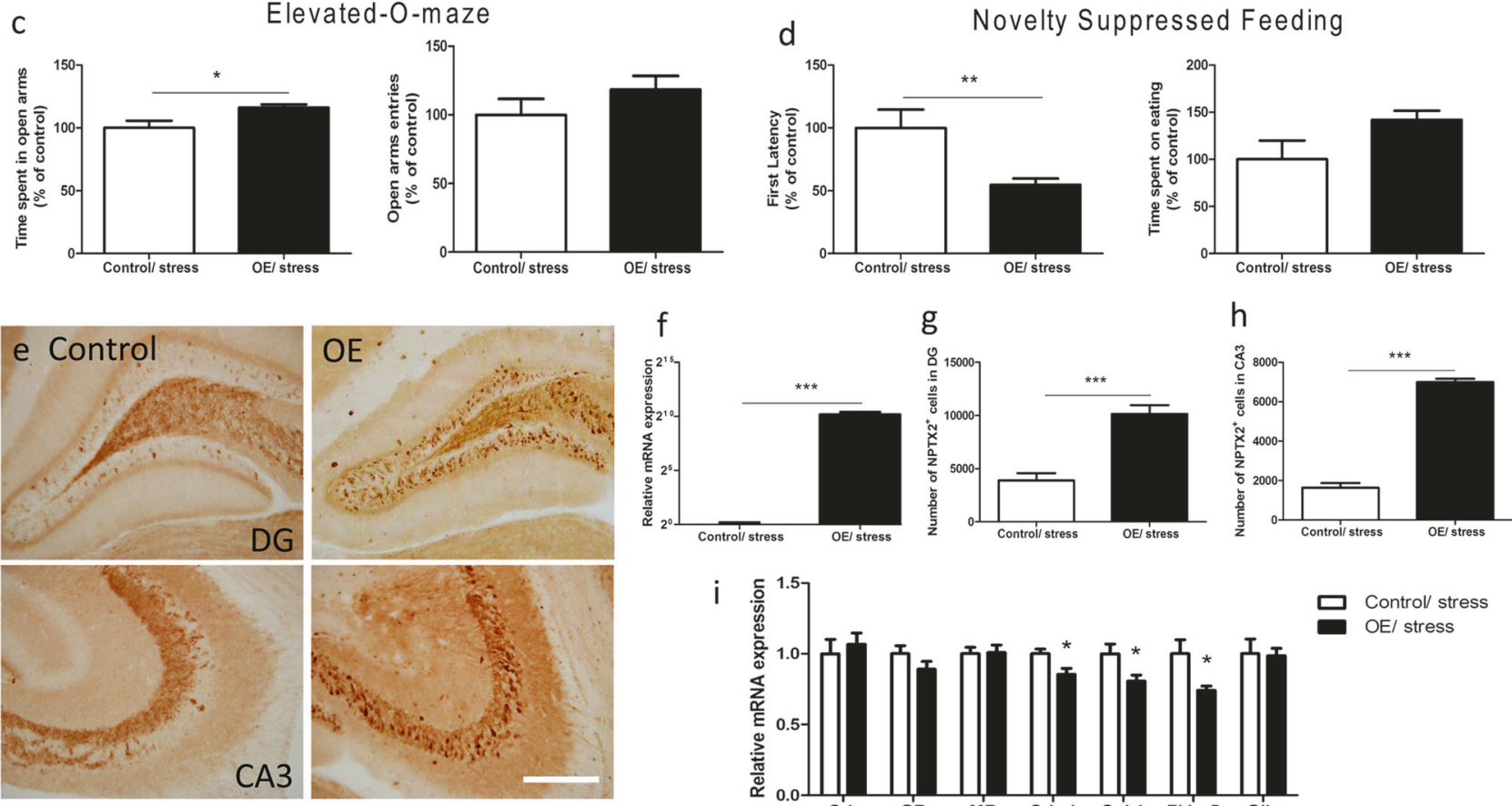

i

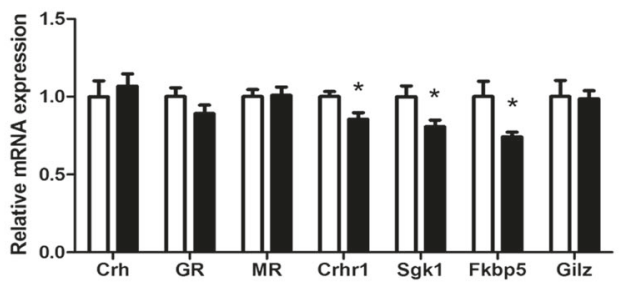

Fig. 6 Overexpression of Nptx2 alleviates stress-induced anxiety and reverses the expression of stress-related genes. a-d Hippocampal Nptx2 overexpression alleviated stress-induced anxiety $(n=10)$. e Immunohistochemistry demonstrated an increase in NPTX2-positive cells in the hippocampus of Nptx2 overexpression mice. $\mathbf{f}$ qPCR confirmed increased mRNA expression of Nptx2 in the hippocampus ( $n=7)$. $\mathbf{g}, \mathbf{h}$ Cell numbers of NPTX2 in DG and CA3 measured by Immunohistochemistry $(n=6)$. $\mathbf{i}$ The expression of glucocorticoid-related genes in the hippocampus of control and Nptx2 overexpression mice after acute stress $(n=7)$. Scale bar represents $200 \mu \mathrm{m}$. Values represent mean \pm SEM. ${ }^{*} p<0.05,{ }^{* *} p<0.01,{ }^{* * *} p<0.001$

influenced its conclusions. Constitutive $\mathrm{Nptx}^{-1-} / \mathrm{Nptxr}^{-1-}$ double $\mathrm{KO}$ mice were used for behavioral tests, but the control mice were half WT and the other half were C57BL/6J mice ordered from Jax lab, non-littermate controls. These confounding environmental factors, including differences in maternal care and colony origin, can impact the assayed measures. In our study, we used littermates for all the experiments, including behavioral tests. Our work provides a multi-pronged approach to clearly delineate the role of Nptx2 in stress and anxiety.

From our previous study we found that the expression of hippocampal Nptx2 is the most significantly upregulated transcript after chronic exercise or antidepressant treatment. Both interventions have been shown increase neurogenesis [1]. Other reports also show antidepressant [13], electroconvulsive seizure [11], or BDNF infusion [12] increases the expression of Nptx2 in the hippocampus. These factors also influence neurogenesis and emotional responses, thus we hypothesize that Nptx2 may play roles in neurogenesis or anxiety. In this study, we show Nptx2 influences cell proliferation, but not neurogenesis. Considerable controversy remains in the literature as to the link between neurogenesis and anxiety. The data we present here suggests that the anxiety phenotype is independent of neurogenesis in Nptx2 $\mathrm{KO}$ mice. It remains possible that some link between the proliferation and the anxiety phenotype exists. Our study does not address the causality between hippocampal cell proliferation and anxiety.

In this study, we found that Nptx2 mediates Crh and GR-related gene expression changes. Crh expression is significantly increased in mice with Nptx2 deletion. Previous study has demonstrated that Crh overexpressing mice exhibit an anxious behavioral state [27]. Conversely, mice lacking $\mathrm{Crhr} 1$ show an impaired stress response and reduced anxiety [29]. We measured expression of GR and its downstream genes. However, we found no significant basal differences between KO and WT mice. One possibility is that the glucocorticoid-related genes we assayed are not activated in the absence of stress. The interaction between genes and environment play an important role in the pathogenesis of anxiety. In acutely stressed mice we detected increased expression levels of GR and its downstream genes, Sgk1 and Fkbp5, in Nptx2 deleted mice. Corticosterone is an adrenal hormone known to activate GR, particularly after stress. We find no difference in the plasma corticosterone levels after stress in our Nptx2 models. Injection of equivalent stress levels of corticosterone causes greater changes in GR-related gene expression in the Nptx2 deleted mice. In combination these data support a model whereby NPTX2 regulates the sensitivity to $\mathrm{GR}$ activation.

Additionally, we found that mice overexpressing Nptx2 were less anxious in response to stress and showed a reversal of the 
stress-induced increases in GR, Sgk1, and Fkbp5 expression. Psychiatric disorders, including anxiety and posttraumatic stress disorder, implicate $F k b p 5$ as a critical gene [30-32]. Several lines of evidence suggest that stress or glucocorticoids increase the expression of Fkbp5 [33, 34]. FKBP5, a co-chaperone of GR, binds to HSP90-GR complex to lower the binding affinity to cortisol, resulting in a decrease of the sensitivity of $G R[30,35]$. In the present study mice without Nptx2 exhibit higher levels of Fkbp5 expression after stress or corticosterone injection. The increased response of Fkbp5 occurs after acute stress in the absence of a difference in corticosterone, suggesting that Nptx2 regulates sensitivity to corticosterone, rather than regulating corticosterone levels per se. With regards to how the changes in Fkbp5 link to the changes in anxiety, it is unclear whether the increased Fkbp5 drives the altered anxiety, or whether the altered anxiety drives changes in Fkbp5 expression. Similarly, hippocampal expression of Sgk1 mRNA is increased in depressed patients and rodents following chronic stress [25]. These results provide molecular evidence that Nptx2 regulates anxiety-related genes after stress. However, in this study the changes of gene expression we detect could result from, rather be the cause of the anxiety phenotype in these mutant mice. Therefore a specific mechanism by which Nptx2 alters stress-induced behavior through Sgk1 and/or Fkbp5 remains undetermined.

In conclusion, our results indicate that hippocampal Nptx2 plays a critical role in modulating anxiety, altering stress sensitivity, and influencing the expression of GR downstream genes. Consequently, we suggest that hippocampal Nptx2 may be a novel target for anxiolytic therapeutics. Further understanding of the underlying cellular mechanisms will help the generation of new management strategies in the treatment of anxiety and stressinduced disorders.

\section{ACKNOWLEDGEMENTS}

We would like to thank Dr. Roderick Carter (University of Edinburg, UK), Dr. Sagiv Shifman (The Hebrew University of Jerusalem, Israel), and Dr. David A. Keays (Research Institute of Molecular Pathology, Austria) for their helpful comments on this manuscript. Funding: This study was supported by grants from the Chang Gung Memorial Hospital (CMRPD1E0312) (to G-JH) and the Ministry of Science and Technology (MOST 105-2320-B-182-040) (to G-JH).

\section{AUTHOR CONTRIBUTIONS}

SC and G-JH conceived the study and wrote the manuscript. SC and PB conducted animal experiments and biochemical studies. C-YT performed blastocyst microinjection. G-JH performed the animal surgery. C-PS made the Nptx2 AAV viral vectors. HL performed RNA-seq experiment. JD contributed reporter mice and provided input on the manuscript.

\section{ADDITIONAL INFORMATION}

Supplementary information accompanies this paper at (https://doi.org/10.1038/ s41386-018-0091-z).

Competing interests: The authors declare no competing interests.

Publisher's note: Springer Nature remains neutral with regard to jurisdictional claims in published maps and institutional affiliations.

\section{REFERENCES}

1. Huang GJ, Ben-David E, Tort Piella A, Edwards A, Flint J, Shifman S. Neurogenomic evidence for a shared mechanism of the antidepressant effects of exercise and chronic fluoxetine in mice. PLoS ONE. 2012;7:e35901.

2. Xiao MF, Xu D, Craig MT, Pelkey KA, Chien CC, Shi Y, et al. NPTX2 and cognitive dysfunction in Alzheimer's Disease. eLife. 2017;6:e23798.

3. Manchia M, Piras IS, Huentelman MJ, Pinna F, Zai CC, Kennedy JL, et al. Pattern of gene expression in different stages of schizophrenia: Down-regulation of NPTX2 gene revealed by a meta-analysis of microarray datasets. Eur Neuropsychopharmacol. 2017:27:1054-63.

4. Hsu YC, Perin MS. Human neuronal pentraxin II (NPTX2): conservation, genomic structure, and chromosomal localization. Genomics. 1995;28:220-7.

5. O'Brien RJ, Xu D, Petralia RS, Steward O, Huganir RL, Worley P. Synaptic clustering of AMPA receptors by the extracellular immediate-early gene product Narp. Neuron. 1999;23:309-23.

6. Sia GM, Beique JC, Rumbaugh G, Cho R, Worley PF, Huganir RL. Interaction of the $\mathrm{N}$-terminal domain of the AMPA receptor GluR4 subunit with the neuronal pentraxin NP1 mediates GluR4 synaptic recruitment. Neuron. 2007;55:87-102.

7. Xu D, Hopf C, Reddy R, Cho RW, Guo L, Lanahan A, et al. Narp and NP1 form heterocomplexes that function in developmental and activity-dependent synaptic plasticity. Neuron. 2003;39:513-28.

8. Chang MC, Park JM, Pelkey KA, Grabenstatter HL, Xu D, Linden DJ, et al. Narp regulates homeostatic scaling of excitatory synapses on parvalbumin-expressing interneurons. Nat Neurosci. 2010;13:1090-7.

9. Pelkey KA, Barksdale E, Craig MT, Yuan X, Sukumaran M, Vargish GA, et al. Pentraxins coordinate excitatory synapse maturation and circuit integration of parvalbumin interneurons. Neuron. 2015;85:1257-72.

10. Alme MN, Wibrand K, Dagestad G, Bramham CR. Chronic fluoxetine treatment induces brain region-specific upregulation of genes associated with BDNFinduced long-term potentiation. Neural Plast. 2007:26496.

11. Reti IM, Baraban JM. Sustained increase in Narp protein expression following repeated electroconvulsive seizure. Neuropsychopharmacology. 2000;23: 439-43.

12. Wibrand K, Messaoudi E, Havik B, Steenslid V, Lovlie R, Steen VM, et al. Identification of genes co-upregulated with Arc during BDNF-induced long-term potentiation in adult rat dentate gyrus in vivo. Eur J Neurosci. 2006;23: 1501-11.

13. Bjartmar L, Alkhori L, Ruud J, Mohammed AH, Marcusson J, Hallbeck M. Longterm treatment with antidepressants, but not environmental stimulation, induces expression of NP2 mRNA in hippocampus and medial habenula. Brain Res. 2010;1328:25-33.

14. Cirulli F, Berry A, Chiarotti F, Alleva E. Intrahippocampal administration of BDNF in adult rats affects short-term behavioral plasticity in the Morris water maze and performance in the elevated plus-maze. Hippocampus. 2004;14:802-7.

15. Takashima Y, Era T, Nakao K, Kondo S, Kasuga M, Smith AG, et al. Neuroepithelial cells supply an initial transient wave of MSC differentiation. Cell. 2007;129: 1377-88.

16. Taniguchi $H$, He M, Wu P, Kim S, Paik R, Sugino K, et al. A resource of Cre driver lines for genetic targeting of GABAergic neurons in cerebral cortex. Neuron. 2011;71:995-1013.

17. Refojo D, Schweizer M, Kuehne C, Ehrenberg S, Thoeringer C, Vogl AM, et al. Glutamatergic and dopaminergic neurons mediate anxiogenic and anxiolytic effects of CRHR1. Science. 2011;333:1903-7.

18. Huang GJ, Edwards A, Tsai CY, Lee YS, Peng L, Era T, et al. Ectopic cerebellar cell migration causes maldevelopment of Purkinje cells and abnormal motor behaviour in Cxcr4 null mice. PLoS ONE. 2014;9:e86471.

19. Quesseveur G, David DJ, Gaillard MC, Pla P, Wu MV, Nguyen HT, et al. BDNF overexpression in mouse hippocampal astrocytes promotes local neurogenesis and elicits anxiolytic-like activities. Transl Psychiatry. 2013;3:e253.

20. Scharfman H, Goodman J, Macleod A, Phani S, Antonelli C, Croll S. Increased neurogenesis and the ectopic granule cells after intrahippocampal BDNF infusion in adult rats. Exp Neurol. 2005;192:348-56.

21. Strohle A, Holsboer F. Stress responsive neurohormones in depression and anxiety. Pharmacopsychiatry. 2003;36:S207-14.

22. Bullitt E. Expression of c-fos-like protein as a marker for neuronal activity following noxious stimulation in the rat. J Comp Neurol. 1990;296:517-30.

23. Davis $M$, Rainnie $D$, Cassell $M$. Neurotransmission in the rat amygdala related to fear and anxiety. Trends Neurosci. 1994;17:208-14.

24. Minelli A, Maffioletti E, Cloninger CR, Magri C, Sartori R, Bortolomasi M, et al. Role of allelic variants of FK506-binding protein 51 (FKBP5) gene in the development of anxiety disorders. Depress Anxiety. 2013;30:1170-6.

25. Anacker C, Cattaneo A, Musaelyan K, Zunszain PA, Horowitz M, Molteni $R$, et al. Role for the kinase SGK1 in stress, depression, and glucocorticoid effects on hippocampal neurogenesis. Proc Natl Acad Sci USA. 2013;110:8708-13.

26. Smythe JW, Murphy D, Timothy C, Costall B. Hippocampal mineralocorticoid, but not glucocorticoid, receptors modulate anxiety-like behavior in rats. Pharmacol Biochem Behav. 1997;56:507-13.

27. Stenzel-Poore MP, Heinrichs SC, Rivest S, Koob GF, Vale WW. Overproduction of corticotropin-releasing factor in transgenic mice: a genetic model of anxiogenic behavior. J Neurosci. 1994;14:2579-84.

28. Solberg LC, Valdar W, Gauguier D, Nunez G, Taylor A, Burnett S, et al. A protocol for high-throughput phenotyping, suitable for quantitative trait analysis in mice. Mamm Genome. 2006;17:129-46. 
NPTX2 is a key component in the regulation...

$S$ Chang et al.

29. Timpl P, Spanagel R, Sillaber I, Kresse A, Reul JM, Stalla GK, et al. Impaired stress response and reduced anxiety in mice lacking a functional corticotropin-releasing hormone receptor 1. Nat Genet. 1998;19:162-6.

30. Binder EB. The role of FKBP5, a co-chaperone of the glucocorticoid receptor in the pathogenesis and therapy of affective and anxiety disorders. Psychoneuroendocrinology. 2009;34:S186-95.

31. Binder EB, Bradley RG, Liu W, Epstein MP, Deveau TC, Mercer KB, et al. Association of FKBP5 polymorphisms and childhood abuse with risk of posttraumatic stress disorder symptoms in adults. JAMA. 2008;299:1291-305.

32. Binder EB, Salyakina D, Lichtner P, Wochnik GM, Ising M, Putz B, et al. Polymorphisms in FKBP5 are associated with increased recurrence of depressive episodes and rapid response to antidepressant treatment. Nat Genet. 2004;36:1319-25.

33. Guidotti G, Calabrese F, Anacker C, Racagni G, Pariante CM, Riva MA. Glucocorticoid receptor and FKBP5 expression is altered following exposure to chronic stress: modulation by antidepressant treatment. Neuropsychopharmacology. 2013;38:616-27.

34. Lee RS, Tamashiro $\mathrm{KL}$, Yang $\mathrm{X}$, Purcell RH, Harvey A, Willour VL, et al. Chronic corticosterone exposure increases expression and decreases deoxyribonucleic acid methylation of Fkbp5 in mice. Endocrinology. 2010;151:4332-43.
35. Scammell JG, Denny WB, Valentine DL, Smith DF. Overexpression of the FK506binding immunophilin FKBP51 is the common cause of glucocorticoid resistance in three New World primates. Gen Comp Endocrinol. 2001;124:152-65.

(i) Open Access This article is licensed under a Creative Commons Attribution 4.0 International License, which permits use, sharing, adaptation, distribution and reproduction in any medium or format, as long as you give appropriate credit to the original author(s) and the source, provide a link to the Creative Commons license, and indicate if changes were made. The images or other third party material in this article are included in the article's Creative Commons license, unless indicated otherwise in a credit line to the material. If material is not included in the article's Creative Commons license and your intended use is not permitted by statutory regulation or exceeds the permitted use, you will need to obtain permission directly from the copyright holder. To view a copy of this license, visit http://creativecommons. org/licenses/by/4.0/.

c The Author(s) 2018 